\title{
Mitochondrial Respiratory Chain and Its Regulatory Elements SIRT1 and SIRT3 Play Important Role in the Initial Process of Energy Conversion after Moxibustion at Local Skin
}

\author{
Ning Zhang, ${ }^{1}$ Na Zhao, ${ }^{1}$ Lu-shuang Xie $\mathbb{D}^{1,2}$ Biao Huang, ${ }^{1}$ Si-rui Lin, ${ }^{1}$ Qun Zhang, \\ Yuan-bing Zhu, ${ }^{1}$ Qiao-feng $W u\left(\mathbb{1},{ }^{1,2,3}\right.$ and Shu-guang $Y u\left({ }^{1}{ }^{1}\right.$ \\ ${ }^{1}$ Acupuncture and Moxibustion School, Chengdu University of Traditional Chinese Medicine, Chengdu, Sichuan 610075, China \\ ${ }^{2}$ Institute of Acupuncture and Homeostasis Regulation, Chengdu University of Traditional Chinese Medicine, Chengdu, \\ Sichuan 610075, China \\ ${ }^{3}$ Acupuncture \& Chronobiology Key Laboratory of Sichuan Province, Chengdu, China
}

Correspondence should be addressed to Qiao-feng Wu; 20052023@cdutcm.edu.cn and Shu-guang Yu; ysg@cdutcm.edu.cn

Received 25 March 2020; Revised 4 June 2020; Accepted 23 July 2020; Published 21 August 2020

Academic Editor: Thomas Efferth

Copyright $\odot 2020$ Ning Zhang et al. This is an open access article distributed under the Creative Commons Attribution License, which permits unrestricted use, distribution, and reproduction in any medium, provided the original work is properly cited.

Objectives. To study how thermal energy is converted after moxibustion at local skin from the view of mitochondrial respiratory chain and its key regulatory elements of sirtuins 1 (SIRT1) and sirtuins 3 (SIRT3). Methods. Two moxibustion temperatures usually used in clinical practice $\left(38^{\circ} \mathrm{C}\right.$ and $\left.46^{\circ} \mathrm{C}\right)$ were applied to Zusanli (ST36) acupoint for 30 minutes in C57BL/6J mice. Local skin samples were harvested at $30 \mathrm{~min}$ and $72 \mathrm{~h}$ after moxibustion intervention, respectively. The activity of mitochondrial respiratory chain complexes I-V was detected by spectrophotometry. The expression of SIRT1 and SIRT3 protein was detected by immunofluorescence staining or western blot. Results. Moxibustion at $38^{\circ} \mathrm{C}$ triggered more significant increase of mitochondrial respiratory chain complexes I-V expression. However, the protein expression of SIRT1 and SIRT 3 at $46^{\circ} \mathrm{C}$ showed more obvious enhancement. In addition, the effect of mitochondrial respiratory chain complexes I-V activity on local skin of ST36 acupoint was more obvious at $30 \mathrm{~min}$ after moxibustion, while the expression of SIRT1 and SIRT3 protein was more significant at $72 \mathrm{~h}$ after moxibustion. Conclusion. Mitochondrial respiratory chain and its key regulatory element proteins SIRT1 and SIRT3 play important role in the initial process of thermal energy conversion stimulated by different moxibustion temperatures in local skin.

\section{Introduction}

Moxibustion is one of the conventional thermal therapies in traditional Chinese medicine, which has been widely used in clinical practice to treat various disorders in China and other Asian countries for thousands of years $[1,2]$. Although many studies have demonstrated that moxibustion has certain effects on the immune system [3], analgesia [4,5], gastrointestinal diseases [6], etc., little is known about the initiation mechanism on conversion process of thermal stimulation induced by moxibustion. In traditional Chinese medicine theory, thermal stimulation produced during moxibustion is considered to be a vital factor in treating diseases. For example, it provides the fundamental basis for exploring the warming-dredging/ promotion of $q i$ and blood theory of moxibustion [7]. A study has demonstrated that the therapeutic effects of moxibustion may be possibly based on the biological effects induced by thermal stimulation [7], which is closely related to the activation of thermoreceptors and nociceptive receptors (transient receptor potential vanilloid, TRPV), heat-sensitive immune cells (Langerhans cells and their major histocompatibility complex II), and heat shock proteins (HSP) at local acupoints [8]. It could be speculated that energy conversion process of thermal stimulation might be the main influencing factor for its subsequent therapeutic effects.

The basic function of mitochondria mitochondrial respiratory chain is to promote the synthesis of adenosine triphosphate (ATP) $[9,10]$, which plays a crucial role in 
energy conversion process. Mitochondria mitochondrial respiratory chain is mainly comprised of four respiratory chain complex enzymes located on the mitochondrial intima, namely, complex I (CI), complex II (CII), complex III (CIII), and complex IV (CIV); these enzymes catalyze the oxidation of biological substrates and the synthesis of ATP. ATP synthetases are also called complex V (CV). The respiratory chain complex enzyme activity can represent the respiratory function of mitochondria directly or indirectly $[11,12]$. Importantly, correlative evidence suggests that mitochondrial respiratory chain complex is also closely related to the thermal stimulation $[13,14]$. SIRT1 and SIRT3 are distributed inside mitochondria [15]. Mitochondrial sirtuins are known to regulate respiratory chain function under physiological conditions and are important regulatory elements of mitochondrial energy flux [16]. Abundant evidence supports the pivotal role of SIRT1 and SIRT3 in the regulation of mitochondrial metabolism and energy homeostasis $[15,17,18]$. Thus, energy conversion related to mitochondria in local skin is one of the important ways to convert thermal energy into biological energy. Nevertheless, changes of mitochondrial respiratory chain complex enzyme activity and their key regulatory element protein SIRT1 and SIRT3 expression at local acupoints after moxibustion treatment are rarely reported.

As a kind of thermal stimulation therapy, many studies have illustrated that moxibustion with different temperatures has different therapeutic effects. During moxibustion, the temperature at local acupoints varies within a range from $34^{\circ} \mathrm{C}$ to $57^{\circ} \mathrm{C}[19,20] .38^{\circ} \mathrm{C}$ is close to the temperature of mild moxibustion treatment, and $46^{\circ} \mathrm{C}$ is close to the temperature of scar moxibustion treatment. Clinical research demonstrated that scar moxibustion temperature at $45^{\circ} \mathrm{C}$ or a little higher is a critical point for the therapeutic effects of moxibustion [21]. For instance, scar moxibustion could significantly reduce the blood lipids level in patients with dyslipidemia, while mild moxibustion temperature at $38^{\circ} \mathrm{C}$ does not have such effect [22]. Our previous animal research observed that the relationship between temperature and effect in moxibustion therapy should have a temperate-specific manner [23]. In chronic inflammatory pain, different moxibustion temperatures (from $37^{\circ} \mathrm{C}$ to $52^{\circ} \mathrm{C}$ ) generated different intensities of analgesic effect: the higher the better $\left(37^{\circ} \mathrm{C}<42^{\circ} \mathrm{C}<47^{\circ} \mathrm{C}<52^{\circ} \mathrm{C}\right)$. In chronic neuropathic pain, stronger analgesic effect was found in moxibustion with temperature $47^{\circ} \mathrm{C}$ or $52^{\circ} \mathrm{C}$ rather than $37^{\circ} \mathrm{C}$ and $42^{\circ} \mathrm{C}$ [23]. Therefore, the present study aims to observe how mitochondrial respiratory chain complexes and their key regulatory element proteins SIRT1 and SIRT3 change in the initial process of moxibustion. Additionally, two usually used moxibustion temperatures $\left(38^{\circ} \mathrm{C}\right.$ and $\left.46^{\circ} \mathrm{C}\right)$ were compared in the study, to illustrate the details of the energy conversion process induced by thermal stimulation at the local skin.

\section{Materials and Methods}

2.1. Animals. A total of 60 male C57BL/6J mice (6-8 weeks) weighing $18 \sim 20 \mathrm{~g}$ were included in the present study. The experimental animals were purchased from Sichuan Dashuo
Experimental Animal Co. Ltd. (license number: SCXK (chuan) 2017-030). Mice were housed in standard animal facilities, in which the room temperature was maintained at $24 \pm 2^{\circ} \mathrm{C}$ and the humidity was $60-80 \%$, with a natural 12 hour light/dark cycles (dark cycle 8:00 PM-8:00 AM). They had free access to water and food and were allowed to acclimate to the housing conditions for 1 week prior to the experiments. Mice were randomly divided into five groups: control group, moxi- $38^{\circ} \mathrm{C}-30 \mathrm{~min}$ group $\left(38^{\circ} \mathrm{C}-30 \mathrm{~min} \cdot \mathrm{MB}\right)$, moxi- $38^{\circ} \mathrm{C}-72 \mathrm{~h}$ group $\left(38^{\circ} \mathrm{C}-72 \mathrm{~h} \cdot \mathrm{MB}\right)$, moxi- $46^{\circ} \mathrm{C}-30 \mathrm{~min}$ group $\left(46^{\circ} \mathrm{C}-30 \mathrm{~min} \cdot \mathrm{MB}\right)$, and $\operatorname{moxi}-46^{\circ} \mathrm{C}-72 \mathrm{~h}$ group $\left(46^{\circ} \mathrm{C}-72 \mathrm{~h} \cdot \mathrm{MB}\right)$ ( $n=12$ group).

All experimental procedures reported here were in accordance with the National Institutes of Health Guide for the Care and Use of Laboratory Animals (NIH Publications no. 80-23) revised in 1996. They also conformed to the Animal Use and Care of Medical Laboratory Animals from the Ministry of Public Health of the People's Republic of China. The study also obtained ethics committee approval from the Committee on Ethical Use of Animals of Chengdu University of Traditional Chinese Medicine (no. 2014-07).

\subsection{Moxibustion Treatment. Moxibustion treatment was} applied to mice in $38^{\circ} \mathrm{C}-30 \mathrm{~min} \cdot \mathrm{MB}, 38^{\circ} \mathrm{C}-72 \mathrm{~h} \cdot \mathrm{MB}, 46^{\circ} \mathrm{C}$ $30 \mathrm{~min} \cdot \mathrm{MB}$, and $46^{\circ} \mathrm{C}-72 \mathrm{~h} \cdot \mathrm{MB}$ group. ST36 (Zusanli) was the selected acupoint, located $2 \mathrm{~mm}$ lateral to the anterior tubercle of the tibia and $4 \mathrm{~mm}$ distal to the knee joint lower point. The location for the acupoint was determined according to Government Channel and Points Standard GB12346-90 of China and the "Veterinary Acupuncture of China." Before the implementation of moxibustion treatment, optimal distance between animal-used moxa sticks $(0.5 \mathrm{~cm}$ diameter, $15 \mathrm{~cm}$ length; Nanyang Hanyi Moxibustion Technology Development Co., Ltd., China) and skin was measured, according to the requirement of temperatures $\left(38^{\circ} \mathrm{C}\right.$ or $\left.46^{\circ} \mathrm{C}\right)$. Moxibustion temperature at ST36 was monitored by a digital thermodetector (WZ-2300R, Xingyi Electronics Company, Hangzhou, China) and stabilized at $38 \pm 1^{\circ} \mathrm{C}$ or $46 \pm 1^{\circ} \mathrm{C}$. The "paperless temperature recorder probe" was placed on the skin surface of moxibustion acupoint in all groups to monitor the real-time temperature [23]. The course of moxibustion treatment was $30 \mathrm{~min}$. There was no treatment applied to mice in control group, except the test of skin temperature of local acupoint in accordance with moxibustion group. Figure 1 shows the temperature curve of local skin during moxibustion.

2.3. Sample Collection. Mice in control group, $38^{\circ} \mathrm{C}$ $30 \mathrm{~min} \cdot \mathrm{MB}$ group, and $46^{\circ} \mathrm{C}-30 \mathrm{~min} \cdot \mathrm{MB}$ group were sacrificed after being anesthetized with $1 \%$ pentobarbital sodium $(3 \mathrm{~mL} / \mathrm{kg}$ ), and skin tissue (approximately $1 \mathrm{~cm} \times 1 \mathrm{~cm})$ in ST36 was harvested. The remaining two groups (moxi- $38^{\circ} \mathrm{C}$ $72 \mathrm{~h}$ group and moxi- $46^{\circ} \mathrm{C}-72 \mathrm{~h}$ group) were sampled 72 hours later; the method was the same as before. Secondly, some of the skin tissues were immersed and fixed in a $4 \%$ paraformaldehyde solution for immunofluorescence, and others were frozen at $-80^{\circ} \mathrm{C}$ for further western blot assay. The mitochondria in skin tissue were extracted by 


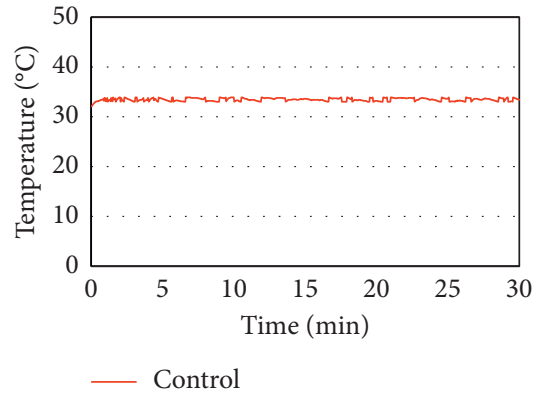

(a)

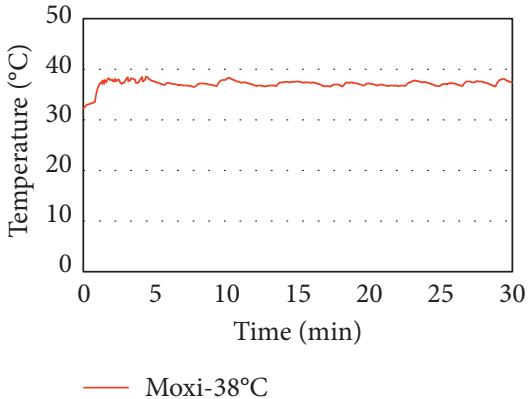

(b)

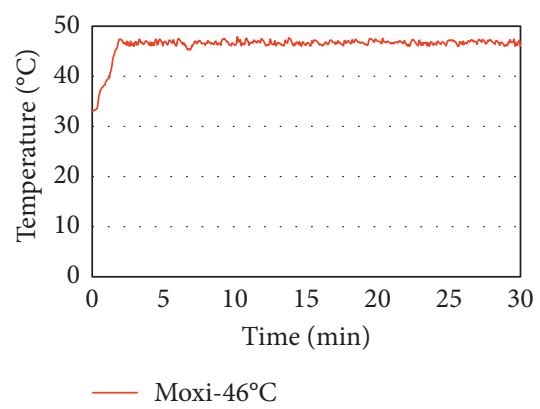

(c)

FIgURE 1: Temperature curve of local skin at ST36 acupoint in mice during moxibustion. (a) Local skin temperature curve of ST36 acupoint in control group was stable at $32 \pm 1^{\circ} \mathrm{C}$, while the temperature in $38^{\circ} \mathrm{C}-\mathrm{MB}$ group was stable at $38 \pm 1^{\circ} \mathrm{C}$ (b) and in $46^{\circ} \mathrm{C}-\mathrm{MB}$ group was stable at $38 \pm 1^{\circ} \mathrm{C}(\mathrm{c})$, respectively, after 2-3 minutes of moxibustion intervention.

mitochondria extraction kit (G006, 50T, Nanjing China); the method of test was in compliance with manufacturer's protocol. The activity of mitochondrial respiratory chain complexes I-V was detected by spectrophotometry. The expression of SIRT1 and SIRT3 in skin tissue was detected by immunofluorescence or western blot.

2.4. Mitochondrial Respiratory Chain Complexes I-V Extraction and Activity Detection. According to the manufacturer's instructions (Mitochondrial Respiratory Chain Complexes I-V Activity Assay Kit, Solarbio Life Science, Beijing, China), every $0.1 \mathrm{~g}$ skin tissue was added to $1 \mathrm{ml}$ extracting solution. The tissue was homogenized in the mortar on the ice. The homogenate was centrifuged at $600 \mathrm{~g}\left(4^{\circ} \mathrm{C}\right)$ for $10 \mathrm{~min}$. The supernatant was transferred into another tube and centrifuged at $11000 \mathrm{~g}\left(4^{\circ} \mathrm{C}\right)$ for $15 \mathrm{~min}$. Next, every $200 \sim 600 \mu \mathrm{l}$ extracting solution was added to the precipitation and broken by the ultrasonic (power 20\%, ultrasonic $5 \mathrm{~s}$ with $10 \mathrm{~s}$ interval, repeated 15 times). Then, the activity of mitochondrial respiratory chain complexes I, II, and V was determined at $340 \mathrm{~nm}$, $605 \mathrm{~nm}$, and $660 \mathrm{~nm}$, respectively; the activity of mitochondrial respiratory chain complexes III and IV was determined at $550 \mathrm{~nm}$, using a spectrophotometer in $1 \mathrm{ml}$ of medium. The enzyme activity is displayed as $\mathrm{nmol} / \mathrm{min} /$ mg protein [24].

2.5. Immunofluorescence Staining. Firstly, samples were deparaffinized in xylene, rehydrated in a series of graded alcohol, and subjected to antigen retrieval. Secondly, sections were blocked with $5 \%$ bovine serum albumin (BSA) in PBS, exposed to $0.5 \%$ Triton X-100 for $2 \mathrm{~h}$, and then incubated with anti-SIRT1 (mouse monoclonal, ab110304, Abcam, UK, 1:200) and anti-SIRT3 (rabbit polyclonal, ab86671, Abcam, UK, $1: 200$ ) overnight at $4^{\circ} \mathrm{C}$. The sections were washed with PBS three times and incubated with secondary antibody (Alexa Fluor 488, bs-0295G-AF488, Bioss, China, 1:200; excitation spectrum $495 \mathrm{~nm}$, emission spectrum $519 \mathrm{~nm}$, cy3, bs-0368R-Cy3, Bioss, China, 1:200; excitation spectrum 552, emission spectrum 570) for $2 \mathrm{~h}$ at $37^{\circ} \mathrm{C}$. Images were captured under laser confocal microscope
(FV1000, Olympus Optical Co., Ltd., Japan). The average optical density of positive expression was quantified by the Image-Pro Plus 6.0 software (Media Cybernetics, USA).

2.6. Western Blot. Western blot analysis was performed as previously described [25]. Whole protein extracts were prepared from mice skin tissue. A total of $20 \mu \mathrm{g}$ of protein from each sample was loaded onto sodium dodecyl sulfate polyacrylamide gel electrophoresis (SDS-PAGE) and separated by electrophoresis. The bands were transferred to a polyvinylidene difluoride membrane. Followed by blocking in 5\% nonfat dry milk in Tris-buffered saline supplemented with $0.1 \%$ Tween 20 for $2 \mathrm{~h}$ at room temperature, the membranes were incubated overnight at $4^{\circ} \mathrm{C}$ with the following antibodies: SIRT1 and SIRT3 primary antibodies (used at 1:800 dilution). The membranes were then incubated with a secondary antibody at $37^{\circ} \mathrm{C}$ for $1 \mathrm{~h}$. Normalization was performed by blotting the same membranes with anti- $\beta$-actin antibody (rabbit monoclonal, ab179467, Abcam. UK, $1: 800$ ). All western blot data were analyzed by Image-Pro Plus 6.0 software.

2.7. Statistical Analysis. All data are expressed as mean \pm standard deviation $(\bar{\chi} \pm \mathrm{SD})$. Normality was checked for all data. Differences between groups were analyzed by one-way analysis of variance (ANOVA) and post hoc test (LeastSignificant Difference, LSD) with GraphPad Prism 7 (GraphPad Prism Software Inc., San Diego, USA). $P<0.05$ was regarded as statistically significant.

\section{Results}

3.1. $38^{\circ} \mathrm{C}-30$ min $M B$ Group Triggered Significant Increase of Mitochondrial Respiratory Chain Complexes I-V Enzyme Expression. Results of "30 min after moxibustion" intervention showed that the activity of mitochondrial respiratory chain complexes I-V in the local skin tissue of ST36 acupoint was elevated after moxibustion intervention. In detail, the activity of complexes $\mathrm{I}-\mathrm{V}$ of mitochondrial respiratory chain in $38^{\circ} \mathrm{C}-30 \mathrm{~min} \cdot \mathrm{MB}$ group significantly increased $(P<0.01)$. In $46^{\circ} \mathrm{C}-30 \mathrm{~min} \cdot \mathrm{MB}$ group, it also 
increased; however, only the activity of mitochondrial respiratory chain complex enzyme III and V showed statistical significance $(P<0.05)$. Additionally, the $46^{\circ} \mathrm{C}-30 \mathrm{~min} \cdot \mathrm{MB}$ group was lower than that in the $38^{\circ} \mathrm{C}-30 \mathrm{~min} \cdot \mathrm{MB}$ group $(P<0.05)$. Results are shown in Figure 2.

Results of " $72 \mathrm{~h}$ after moxibustion" intervention showed that, except for the activity of complex I in $38^{\circ} \mathrm{C}-72 \mathrm{~h} \cdot \mathrm{MB}$ group $(P<0.05)$, the activity of complexes $\mathrm{I}-\mathrm{V}$ was slightly higher than that in the control group; however, there was no significant difference $(P>0.05)$. Furthermore, no statistical significance was found between $38^{\circ} \mathrm{C}-72 \mathrm{~h} \cdot \mathrm{MB}$ group and $46^{\circ} \mathrm{C}-72 \mathrm{~h} \cdot \mathrm{MB}$ group.

On the whole, in $38^{\circ} \mathrm{C}-\mathrm{MB}$ group, the activity of mitochondrial respiratory chain complexes I-V in the local skin tissue of ST36 acupoints in "72 h after moxibustion" intervention was significantly decreased compared to that in the "30 minutes after moxibustion" intervention, but it was still slightly higher than that in the control group. In the $46^{\circ} \mathrm{C}-\mathrm{MB}$ group, the activity of mitochondrial respiratory chain complexes I-V also decreased, but it was not statistically significant. Additionally, the $38^{\circ} \mathrm{C}-\mathrm{MB}$ group had a continuously higher level than that in the $46^{\circ} \mathrm{C}-\mathrm{MB}$ group.

\section{2. $46^{\circ} \mathrm{C}-72 \mathrm{~h} \mathrm{MB}$ Group Triggered Obvious Enhancement of the SIRT1 and SIRT3 Protein Expression.} Immunofluorescence staining and analysis showed that, compared with the control group, the expression of SIRT1 and SIRT3 protein in ST36 acupoint local skin tissue indicated increasing trend after moxibustion intervention. In $46^{\circ} \mathrm{C}-30 \mathrm{~min} \cdot \mathrm{MB}$ group and $46^{\circ} \mathrm{C}-72 \mathrm{~h} \cdot \mathrm{MB}$ group, the expression of SIRT1 and SIRT3 in local skin tissue of ST36 acupoint was significantly enhanced $(P<0.01)$. In $38^{\circ} \mathrm{C}$ $30 \mathrm{~min} \cdot \mathrm{MB}$ group and $38^{\circ} \mathrm{C}-72 \mathrm{~h} \cdot \mathrm{MB}$ group, it also increased but exhibited no statistical significance $(P>0.05)$. Additionally, the expression of SIRT1 and SIRT3 protein in $38^{\circ} \mathrm{C}$ $\mathrm{MB}$ group was lower than that in the $46^{\circ} \mathrm{C}-\mathrm{MB}$ group with statistical significance $(P<0.05, P<0.01)$. Results are shown in Figures 3 and 4.

Western blots and analysis showed that the expression of SIRT1 and SIRT3 protein in ST36 acupoint in local skin tissue also indicated increasing trend after moxibustion intervention. The expression of SIRT3 protein in local skin tissue of ST36 acupoint showed the same trend as that of immunofluorescence staining results $(P<0.01)$. However, compared with the control group, the increase of SIRT1 protein expression in $38^{\circ} \mathrm{C}-30 \mathrm{~min} \cdot \mathrm{MB}$ group and $38^{\circ} \mathrm{C}$ $72 \mathrm{~h} \cdot \mathrm{MB}$ group was not statistically significant $(P>0.05)$, and the expression of SIRT1 protein in $46^{\circ} \mathrm{C}-30 \mathrm{~min} \cdot \mathrm{MB}$ group and $46^{\circ} \mathrm{C}-72 \mathrm{~h} \cdot \mathrm{MB}$ group was significantly enhanced $(P<0.05)$. In particular, $46^{\circ} \mathrm{C}-72 \mathrm{~h} \cdot \mathrm{MB}$ group kept a higher level than that in $46^{\circ} \mathrm{C}-30 \mathrm{~min} \cdot \mathrm{MB}$ group. Results are shown in Figures 3 and 4.

\section{Discussion}

The effect of moxibustion, as a kind of warm stimulation, is closely related to the quantity and time of moxibustion as well as the temperature and density of moxibustion.
Different moxibustion temperatures have different effects on the local microenvironment, such as blood flow, cell types and numbers, cytokines, receptors, and HSP [22, 26-28]. It is considered that suitable temperature of thermal stimulation was $40-41^{\circ} \mathrm{C}$ in human body since this temperature could increase the content of ATP, adenosine diphosphate (ADP), and adenosine monophosphate (AMP), enhance the cell activity, and improve the energy metabolism level of the body [26]. However, Zhu [21] reported that the moxibustion effect below $40^{\circ} \mathrm{C}$ was basically invalid, while the valid moxibustion temperature must reach or exceed $45^{\circ} \mathrm{C}$, because it could not only activate the C-type nociceptor, but also activate the transient receptor potential vanillic acid receptor subtype I (TRPV1). Li et al. [29] compared the inhibitory effect of different moxibustion temperatures $\left(40^{\circ} \mathrm{C}, 42^{\circ} \mathrm{C}, 44^{\circ} \mathrm{C}, 46^{\circ} \mathrm{C}, 48^{\circ} \mathrm{C}, 50^{\circ} \mathrm{C}, 52^{\circ} \mathrm{C}\right)$ on the activation of wide dynamic neurons in spinal dorsal horn induced by nociceptive colon-rectum distention. They found that $46^{\circ} \mathrm{C} \sim 48^{\circ} \mathrm{C}$ range was the best active temperature. Researchers also found that moxibustion at $46^{\circ} \mathrm{C}$ can significantly increase the number of mast cells (MC) in the acupoint area, followed by promoting MC degranulation, secreting chemical media, and participating in local immune response [30]. Our previous study also found that there was a temperature difference in the effect of moxibustion on the shape, distribution, and quantity of Langerhans cells at the acupoint area [28]. Therefore, revealing the details of moxibustion temperature is an important way to understand the initial mechanism of moxibustion.

In the present study, we found that different moxibustion temperatures had variable effects on the mitochondrial respiratory chain complexes I-V activity and their key regulatory element proteins SIRT1 and SIRT3 expression. This may provide a basis for energy conversion from thermal stimulation to biological effect by moxibustion intervention.

The results showed that both moxibustion at $38^{\circ} \mathrm{C}$ and $46^{\circ} \mathrm{C}$ can promote the expression of mitochondrial respiratory chain complex activity in acupoints, indicating that thermal stimulation can increase the production of mitochondrial ATP and provide energy for the body. The basic function of the mitochondrial respiratory chain is to convert the reduction potential energy generated by the substrate redox process into proton electrochemical energy, and the proton electrochemical energy can be converted into ATP $[9,10]$. It is reported that $95 \%$ of the ATP in organisms comes from oxidative phosphorylation (OXPHOS) of mitochondria, and the level of mitochondrial OXPHOS affects the maintenance of energy in the body. Importantly, the mitochondrial respiratory chain plays an important role in the process of mitochondrial OXPHOS [31]. Our previous study found that there was enrichment of OXPHOS signal pathway at ST36 acupoint after moxibustion [32] and there was also a large amount of ATP release [33]. Therefore, OXPHOS may be the upstream biological process of local ATP at acupoints involved in moxibustion. Additionally, we found that moxibustion temperature of $38^{\circ} \mathrm{C}$ is better than $46^{\circ} \mathrm{C}$ on the basis of mitochondrial respiratory chain complex activity, and the immediate effect after moxibustion 


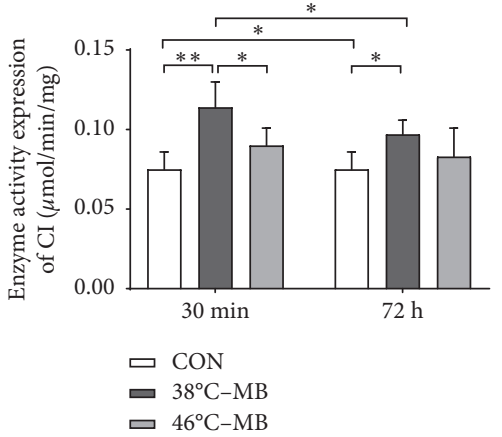

(a)

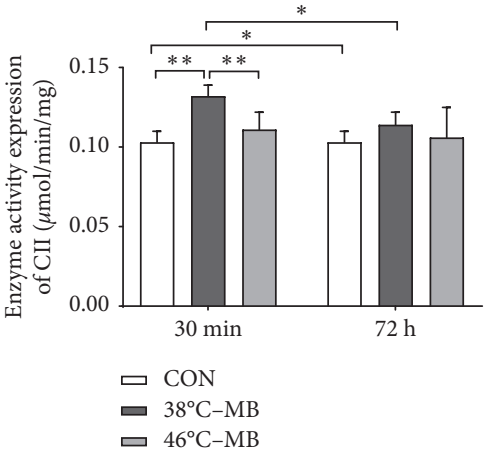

(b)

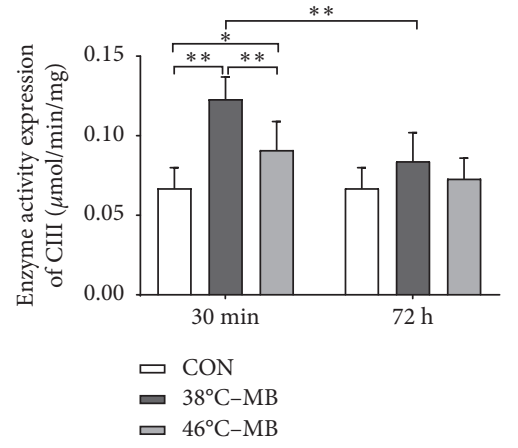

(c)

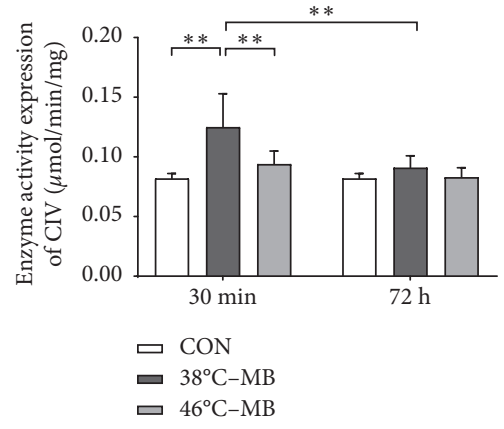

(d)

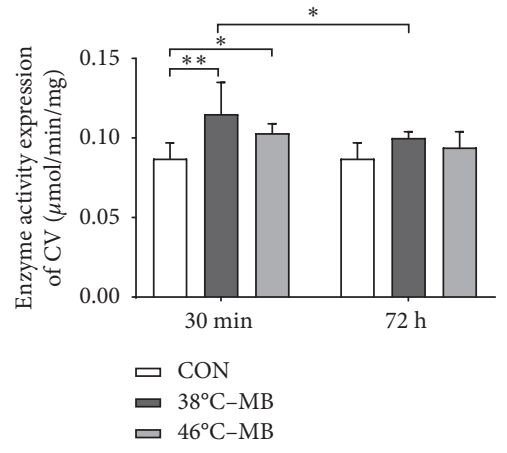

(e)

FIgURe 2: Comparison of the activity of mitochondrial respiratory chain complexes I-V enzyme after moxibustion intervention. (a-e) The expression of mitochondrial respiratory chain complexes I-V enzyme activity in ST36 local skin tissue, at 30 minutes and 72 hours after moxibustion intervention with different temperatures $\left({ }^{*} P<0.05,{ }^{* *} P<0.01, n=6\right)$. MB: moxibustion; CI: complex I; CII: complex II; CIII: complex III; CIV: complex IV; CV: complex V.

is better than the continuous effect (" 30 minutes after moxibustion" is better than " 72 hours after moxibustion"). This seems to explain our previous study results which showed that local ATP increased significantly in complete Freund's adjuvant-induced chronic pain model and corresponding analgesic effect reached the peak after 45 min's moxibustion [34]. We speculated that the ATP level of the local analgesic substance in the acupoints may be related to the increase of the activity of mitochondrial respiratory chain complex enzyme after moxibustion.

Compared with $38^{\circ} \mathrm{C}-\mathrm{MB}$ group, lower activity of mitochondrial respiratory chain complex and higher expression of SIRT1 and SIRT3 protein were found in $46^{\circ} \mathrm{C}-\mathrm{MB}$ group. Studies have reported that higher temperatures $\left(<52^{\circ} \mathrm{C}\right)$ cause increased membrane permeability of mitochondria, prompting exogenous $\mathrm{H}_{2} \mathrm{O}_{2}$ to enter the mitochondria, which results in accumulation of $\mathrm{H}_{2} \mathrm{O}_{2}$ in the mitochondria. However, higher mitochondrial $\mathrm{H}_{2} \mathrm{O}_{2}$ levels are always accompanied by lower electron transport chain complex I activity [35]. Thus, inhibition of complex I enzyme activity could lead to its electron transport barrier and then induce decreased activity of other complexes, which might be an important reason why the local mitochondrial respiratory chain complex activity expression in $46^{\circ} \mathrm{C}-\mathrm{MB}$ group is lower than that in $38^{\circ} \mathrm{C}-\mathrm{MB}$ group. However, further investigation is needed. SIRT3 is called "mitochondrial sirtuins" given its mitochondrial location [36].
Overexpression of SIRT3 in cells affects expression of genes involved in mitochondrial function. In light of the central role of mitochondria in many cellular processes, SIRT3 has functions aside from metabolism, for example, glycolipid metabolism, tricarboxylic acid cycle, electron transfer chain, oxidative stress, and apoptosis [37]. Furthermore, Aquilano et al. found that SIRT1 is also distributed inside mitochondria [15]. Studies have shown that the activity of SIRT1 can be enhanced with increasing temperature [38]; importantly, its activity is crucial for maintaining mitochondrial integrity and energy metabolism, alternatively, regulating cell functions including cell metabolism and cellular stress response $[39,40]$. Briefly, it could be proposed that SIRT1 and SIRT3 protein may play important role in the mitochondrial integrity function after moxibustion treatment.

Studies have suggested that thermal stimulation with relatively higher temperature can induce the production of reactive oxygen species, promote the oxidation of cytochrome $\mathrm{C}$, enhance the permeability of mitochondrial membrane and then promote its release into the cytoplasm, and increase the intracellular $\mathrm{pH}$ value, which makes the thermal control ion channel of TRPV1 open. The activation of TRPV1 can result in extracellular $\mathrm{Ca}^{2+}$ influx, accompanied by a small amount of $\mathrm{Mg}^{2+}, \mathrm{K}^{+}$, and $\mathrm{Na}^{+}$, which causes the release of neurotransmitters, as well as a series of biological phenomena such as cell apoptosis and 

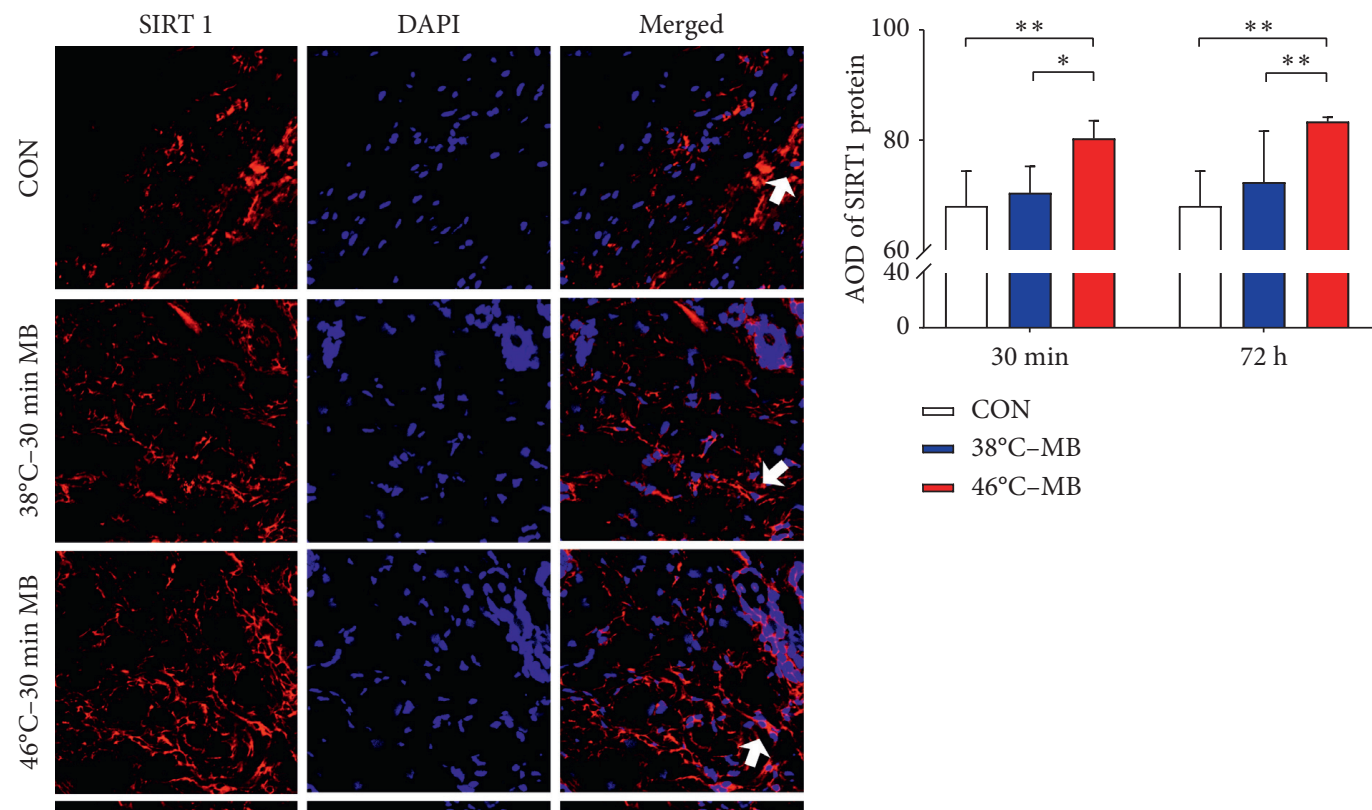

$$
\begin{aligned}
& \text { च } \mathrm{CON} \\
& =38^{\circ} \mathrm{C}-\mathrm{MB} \\
& \text { ש } 46^{\circ} \mathrm{C}-\mathrm{MB}
\end{aligned}
$$
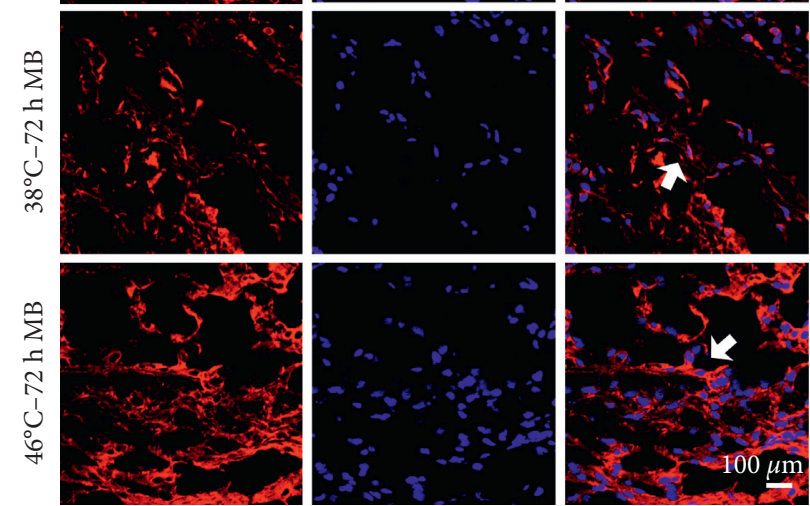

(a)
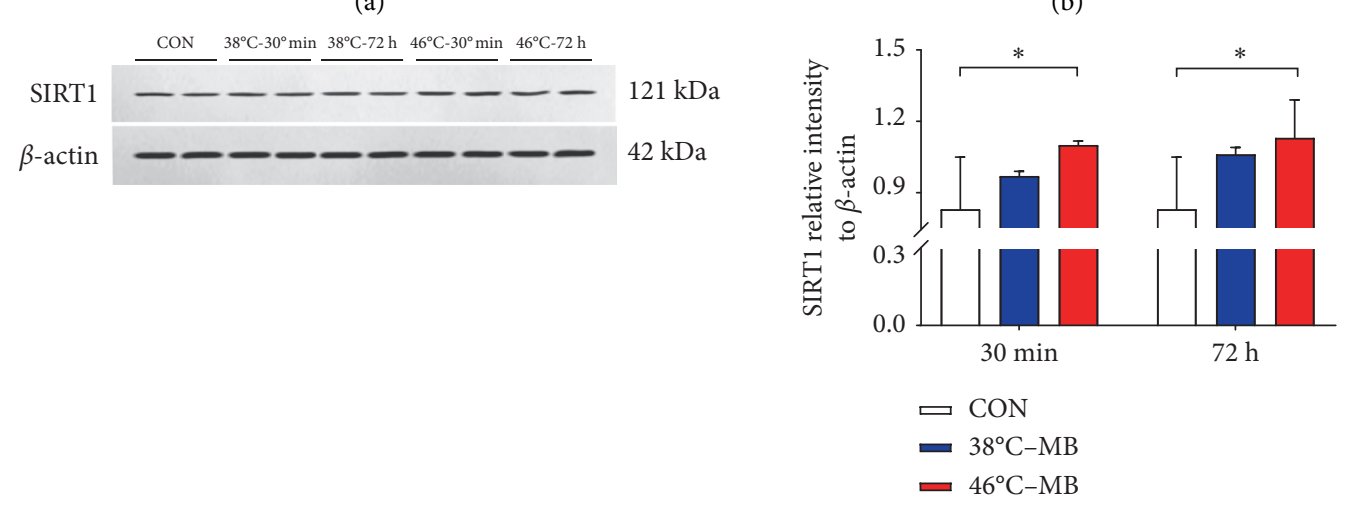

FIgURE 3: Different moxibustion temperatures have certain effect on SIRT1 protein expression in ST36 acupoint local skin tissue. (a-b) Representative labeling (a) and analysis (b) of SIRT1 protein expression in ST36 local skin tissue at 30 minutes and 72 hours after moxibustion intervention. Red SIRT1, blue DAPI nuclear staining $\left({ }^{*} P<0.05,{ }^{* *} P<0.01, n=6\right.$ /group). White arrow highlights the positive expression of SIRT1 protein. (c-d) Western blots (c) and analysis (d) showing the upregulation of SIRT1 protein expression in ST36 local skin tissue at 30 minutes and 72 hours after moxibustion intervention $\left({ }^{*} P<0.05,{ }^{* *} P<0.01, n=3\right.$ /group).

mitochondrial structure and function disorders [41]. It is possible that a similar mechanism might be involved when moxibustion is at $46^{\circ} \mathrm{C}$. We also found that the activity of mitochondrial respiratory chain complexes $\mathrm{I}-\mathrm{V}$ in the local skin tissue of ST36 acupoint in " $72 \mathrm{~h}$ after moxibustion" intervention was continuously at a higher level than that in the control group, and the protein expression of SIRT1 and SIRT3 was higher than that of " 30 minutes after moxibustion" intervention. Therefore, the therapeutic effect of moxibustion might persist even after 72 hours. 

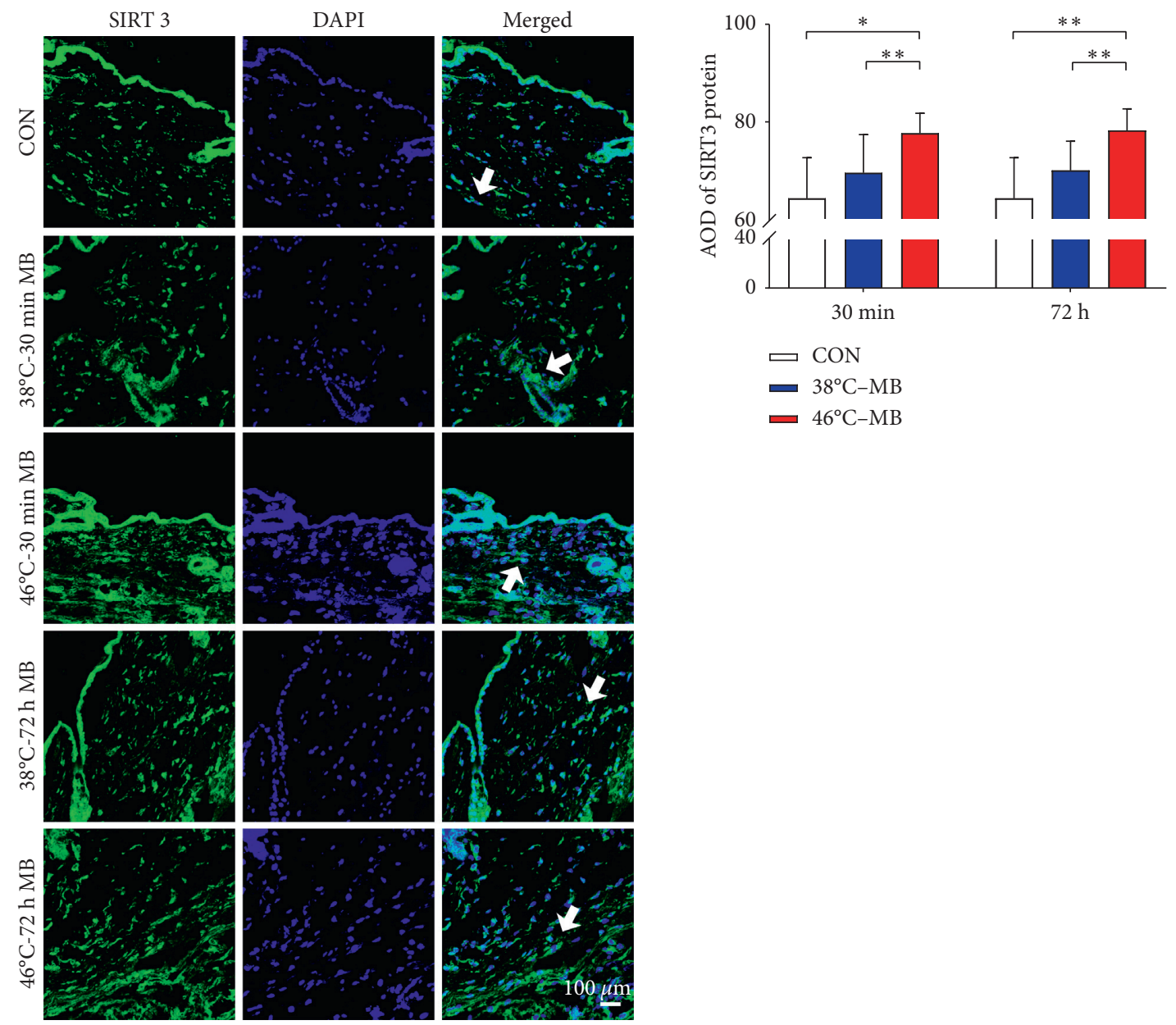

(a)

$$
\begin{aligned}
& =\mathrm{CON} \\
& =38^{\circ} \mathrm{C}-\mathrm{MB} \\
& \square 46^{\circ} \mathrm{C}-\mathrm{MB}
\end{aligned}
$$

(b)
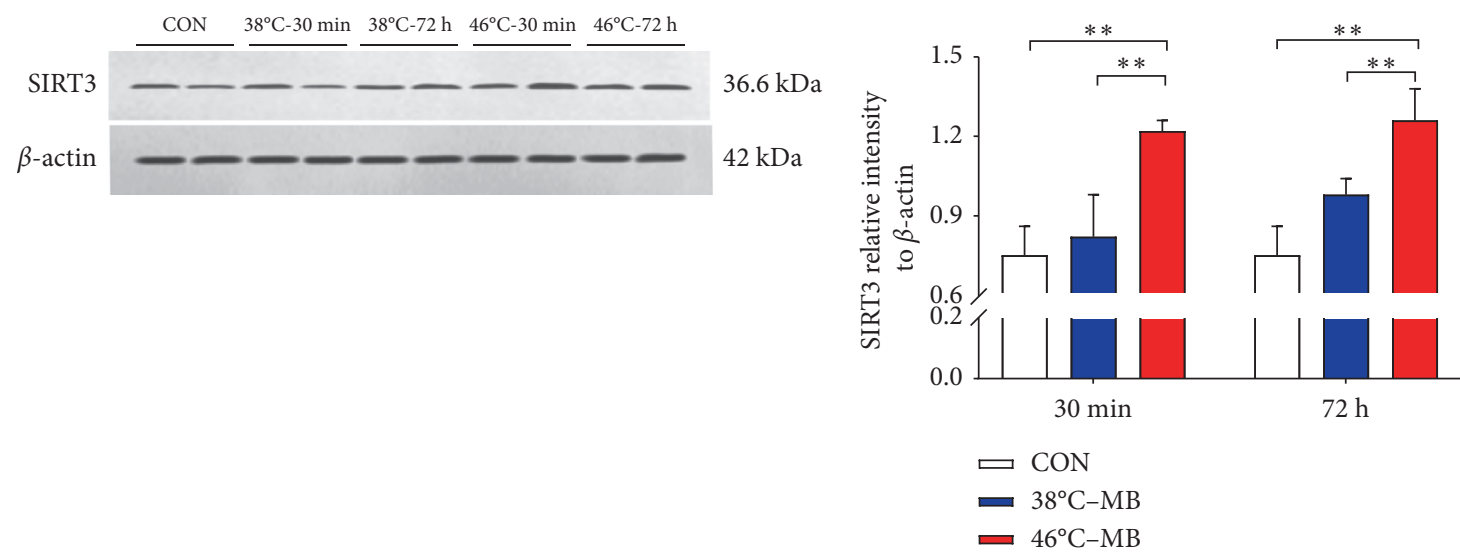

(c)

(d)

FIGURE 4: Different moxibustion temperatures have certain effect on SIRT3 protein expression in ST36 acupoint local skin tissue. (a-b) Representative labeling (a) and analysis (b) of SIRT3 protein expression in ST36 local skin tissue at 30 minutes and 72 hours after moxibustion intervention. Green SIRT3, blue DAPI nuclear staining $\left({ }^{*} P<0.05,{ }^{* *} P<0.01, n=6 /\right.$ group$)$. White arrow highlights the positive expression of SIRT3 protein. (c-d) Western blots (c) and analysis (d) showing the upregulation of SIRT3 protein expression in ST36 local skin tissue at 30 minutes and 72 hours after moxibustion intervention $\left({ }^{*} P<0.05,{ }^{* *} P<0.01, n=3 /\right.$ group $)$.

ST36 is one of the most used acupoints in clinical practice of traditional Chinese medicine, as well as basic and clinical studies. Studies have reported that acupuncture or moxibustion at ST36 acupoint has good effects on knee osteoarthritis [42], gastrointestinal diseases [43, 44], anesthesia [45], anorexia and improving quality of life [46], etc. Electroacupuncture at ST36 acupoint can effectively inhibit lipopolysaccharide-induced multiple peritonitis in mice 
[47]. ST36 was also chosen to investigate the mechanism of moxibustion analgesic effect in our previous studies [23, 48, 49]. Moxibustion at ST36 acupoint can effectively increase the expression of HSP in cardiac muscle, brain tissue, gastric mucosa, and other distal tissues and organs in healthy rats [50]. According to the theory of Chinese medicine, even under healthy state, moxibustion at ST36 is often used for healthcare and enhances the body status. For instance, in ancient Chinese medical practices, it is associated with the extension of lifespan.

Hence, based on the evidence provided in current and previous studies, we could speculate that the enzymatic activities of mitochondrial respiratory chain complexes and their key regulatory element proteins SIRT1 and SIRT3 expression on local acupoint skin may participate in the initial process of energy conversion after moxibustion. These results implied that moxibustion at $38^{\circ} \mathrm{C}$ and $46^{\circ} \mathrm{C}$ will have different initial energy conversion at local skin which might induce different biological effect.

In future study, we should use antagonists to block the upregulation of "sirtuins" to verify whether the upregulation of "sirtuins" leads to upregulation of respiratory chain complex expression. More specific studies on, for example, mitochondrial marker enzymes should be detected to verify the general and specific effect on mitochondria of respiratory chain enzymes. Additionally, we did not set the intermediate time point group. It is necessary to conduct further research on the time course correlation of mitochondrial respiratory chain complexes and their regulatory element proteins SIRT1 and SIRT3 after thermal stimulation by observing the changes at several time points after moxibustion.

\section{Conclusion}

Mitochondrial respiratory chain is involved in the initial process of thermal energy conversion stimulated by different moxibustion temperatures in local skin. It could be proposed that SIRT1 and SIRT3 protein in local skin may play important role in the mitochondrial integrity function after moxibustion treatment.

\section{Data Availability}

The data used to support the findings of this study are available from the corresponding author upon request.

\section{Conflicts of Interest}

The authors declare that they have no conflicts of interest.

\section{Authors' Contributions}

Ning Zhang and Qiao-feng Wu wrote the paper; Shu-guang $\mathrm{Yu}$ and Qiao-feng $\mathrm{Wu}$ conceived and designed the experiments; Na Zhao, Ning Zhang, Lu-shuang Xie, Biao Huang, Si-rui Lin, Qun Zhang, and Yuan-bing Zhu performed the experiments; Ning Zhang, Na Zhao, and Qiao-feng Wu analyzed the images and the data. All the authors approved the final version of the manuscript. Ning Zhang and $\mathrm{Na}$ Zhao contributed equally to this work.

\section{Acknowledgments}

This work was supported by the National Key R\&D Program of China (no. 2019YFC1709001); National Natural Science Foundation of China (nos. 81873383, 81873386, and 81704160); fund of Sichuan Science and Technology Program, China (no. 2018JY0095); and fund of Sichuan Provincial Department of Education, China (no. 18TD0018).

\section{References}

[1] H. W. Zhang, Z. X. Lin, F. Cheung, W. C.-S. Cho, and J.-L. Tang, "Moxibustion for alleviating side effects of chemotherapy or radiotherapy in people with cancer," The Cochrane Database of Systematic Reviews, vol. 11, Article ID Cd010559, 2018.

[2] M. Y. Lim, J. Huang, and B. Zhao, "Standardisation of moxibustion: challenges and future development," $\mathrm{Acu}$ puncture in Medicine, vol. 33, no. 2, pp. 142-147, 2015.

[3] S. G. Yu, X. H. Jing, Y. Tang et al., "Acupuncture and moxibustion and immunity: the actuality and future," Acupuncture Research, vol. 43, no. 12, pp. 747-753, 2018.

[4] M. Yang, X. Chen, L. Bo et al., "Moxibustion for pain relief in patients with primary dysmenorrhea: a randomized controlled trial," PLoS One, vol. 12, no. 2, Article ID e0170952, 2017.

[5] M. S. Lee, T.-Y. Choi, J. W. Kang, B.-J. Lee, and E. Ernst, "Moxibustion for treating pain: a systematic review," The American Journal of Chinese Medicine, vol. 38, no. 5, pp. 829-838, 2010.

[6] J.-M. Zhao, L.-Y. Wu, H.-R. Liu et al., "Factorial study of moxibustion in treatment of diarrhea-predominant irritable bowel syndrome," Gastroenterology Clinics of North America, vol. 20, no. 37, pp. 13563-13572, 2014.

[7] K.-y. Huang, S. Liang, L. Lu, P. J. Morgan, and J.-b. Zhang, “To understand moxibustion from the biological effect of local thermal stimulation," World Journal of Acupuncture-Moxibustion, vol. 26, no. 3, pp. 31-48, 2016.

[8] K. Zhang, S. Liang, Z. Sun, and J. Zhang, "Startup mechanism of moxibustion warming and dredging function," Chinese Acupuncture \& Moxibustion, vol. 37, no. 7, pp. 1023-1026, 2017.

[9] S. Cogliati, I. Lorenzi, G. Rigoni, F. Caicci, and M. E. Soriano, "Regulation of mitochondrial electron transport chain assembly," Journal of Molecular Biology, vol. 430, no. 24, pp. 4849-4873, 2018.

[10] L. Holper, D. Ben-Shachar, and J. Mann, "Multivariate metaanalyses of mitochondrial complex I and IV in major depressive disorder, bipolar disorder, schizophrenia, Alzheimer disease, and Parkinson disease," Neuropsychopharmacology, vol. 44, no. 5, pp. 837-849, 2019.

[11] P. Rustin, D. Chretien, T. Bourgeron et al., "Biochemical and molecular investigations in respiratory chain deficiencies," Clinica Chimica Acta, vol. 228, no. 1, pp. 35-51, 1994.

[12] R. G. Feichtinger, M. Oláhová, Y. Kishita et al., "Biallelic C1QBP mutations cause severe neonatal-, childhood-, or later-onset cardiomyopathy associated with combined respiratory-chain deficiencies," The American Journal of Human Genetics, vol. 101, no. 4, pp. 525-538, 2017.

[13] C. A. Downs and S. A. Heckathorn, "The mitochondrial small heat-shock protein protects NADH:ubiquinone oxidoreductase of the electron transport chain during heat stress in plants," FEBS Letters, vol. 430, no. 3, pp. 246-250, 1998. 
[14] Q.-L. Zhao, Y. Fujiwara, and T. Kondo, "Mechanism of cell death induction by nitroxide and hyperthermia," Free Radical Biology and Medicine, vol. 40, no. 7, pp. 1131-1143, 2006.

[15] K. Aquilano, P. Vigilanza, S. Baldelli, B. Pagliei, G. Rotilio, and M. R. Ciriolo, "Peroxisome proliferator-activated receptor $\gamma$ Co-activator $1 \alpha$ (PGC- $1 \alpha)$ and sirtuin 1 (SIRT1) reside in mitochondria," Journal of Biological Chemistry, vol. 285, no. 28, pp. 21590-21599, 2010.

[16] A. B. Potthast, T. Heuer, S. J. Warneke, and A. M. Das, "Alterations of sirtuins in mitochondrial cytochrome c-oxidase deficiency," PLoS One, vol. 12, no. 10, Article ID e0186517, 2017.

[17] Y.-T. Wu, H.-C. Lee, C.-C. Liao, and Y.-H. Wei, "Regulation of mitochondrial $\mathrm{F}_{\mathrm{o}} \mathrm{F}_{1}$ ATPase activity by Sirt3-catalyzed deacetylation and its deficiency in human cells harboring 4977 bp deletion of mitochondrial DNA," Biochimica et Biophysica Acta (BBA)-Molecular Basis of Disease, vol. 1832, no. 1, pp. 216-227, 2013.

[18] B.-H. Ahn, H.-S. Kim, S. Song et al., "A role for the mitochondrial deacetylase Sirt3 in regulating energy homeostasis," Proceedings of the National Academy of Sciences, vol. 105, no. 38, pp. 14447-14452, 2008.

[19] J. Jiang, X. Wang, X. Wu, and Z. Yu, “Analysis of factors influencing moxibustion efficacy by affecting heat-activated transient receptor potential vanilloid channels," Journal of Traditional Chinese Medicine, vol. 36, no. 2, pp. 255-260, 2016.

[20] J. J. Xin, Y. S. Su, Z. K. Yang et al., "Effects of electroacupuncture and regional thermal stimulation at "Zusanli" (ST 36) on pain thresholds of TRPV 1 knock-out mice," Acupuncture Research, vol. 37, no. 6, pp. 431-439, 2012.

[21] B. Zhu, "On the considerations about heating materials and temperature of moxibustion in clinical practice," Acupuncture Research, vol. 43, no. 2, pp. 63-67, 2018.

[22] J. Gao, G. Wang, and L. Wang, "Clinical study on the effect of moxibustion at different temperatures on regulating lipid and dredging pulse mediated by TRPV1," Li Shizhen Medicine and Materia Medica Research, vol. 26, no. 9, pp. 2182-2184, 2015.

[23] W. Zhou, R. Lei, C. Zuo et al., "Analgesic effect of moxibustion with different temperature on inflammatory and neuropathic pain mice: a comparative study," Evidence-Based Complementary and Alternative Medicine, vol. 2017, Article ID 4373182, 8 pages, 2017.

[24] M. Wang, Y. Zhang, M. Xu et al., "Roles of TRPA1 and TRPV1 in cigarette smoke-induced airway epithelial cell injury model," Free Radical Biology and Medicine, vol. 134, pp. 229-238, 2019.

[25] J. Sun, H. Zhang, C. Wang et al., "Regulating the balance of Th17/treg via electroacupuncture and moxibustion: an ulcerative colitis mice model based study," Evidence-Based Complementary and Alternative Medicine, vol. 2017, Article ID 7296353, 13 pages, 2017.

[26] Z.-h. Yang, J.-1. Xiao, X. Yang, J. Cui, and B. Chen, "Effects of external thermal stimulation on the energy substance of human umbilical venous endothelial cells," China Journal of Traditional Chinese Medicine and Pharmacy, vol. 30, no. 1, pp. 255-258, 2015.

[27] W. Y. Zong, Q. Wang, and J. Cai, "Effects of different moxibustion temperatures on skin morphology and mast cells of blood lipid and shenque point in mice with hyperlipidemia," Li Shizhen Medicine and Materia Medica Research, vol. 28, no. 12, pp. 3020-3022, 2017.

[28] H. Yang, Effects of Different Temperature Moxibustion on Local Langerhans Cells in Mouse Acupoints and its Correlation with TRPV1, Chengdu University of Traditional Chinese Medicine, Chengdu, China, 2017.

[29] L. Li, P. J. Rong, and L. L. Yu, "Study on spinal cord mechanisms of inhibitory effect of moxibustion on nociceptive expansion of rectal colon," Chinese Journal of Basic Medicine in TCM, vol. 17, no. 10, pp. 1140-1145, 2011.

[30] G. Wang, Y. Wang, K. Lu, L. Zhong, and L. Wang, "Effects of different moxibustion temperature on cholesterol and skin around "Shenque" (CV 8) in mice with hyperlipidemia," Chinese Acupuncture \& Moxibustion, vol. 36, no. 1, pp. 59-63, 2016.

[31] K. M. Davies, M. Strauss, B. Daum et al., "Macromolecular organization of ATP synthase and complex I in whole mitochondria," Proceedings of the National Academy of Sciences, vol. 108, no. 34, pp. 14121-14126, 2011.

[32] L. Zhao, The Influence of Moxibustion at ST36 on the Expression of Genes Related Oxidative Phosphorylation in Adjuvant Arthritis Rats, Chengdu University of TCM, Chengdu, China, 2013.

[33] Y.-p. Fan, Local ATP Mediates Anti-nociceptive Effects of Moxibustion, Chengdu University of traditional Chinese Medicine, Chengdu, China, 2014.

[34] L.-p. Hu, Adenosine A1 Receptors Mediate Anti-Nociceptive Effects of Moxibustion, Chengdu University of Traditional Chinese Medicine, Chengdu, China, 2016.

[35] R. Hastie and M. Lappas, "The effect of pre-existing maternal obesity and diabetes on placental mitochondrial content and electron transport chain activity," Placenta, vol. 35, no. 9, pp. 673-683, 2014.

[36] G. Liu, S.-H. Park, M. Imbesi et al., "Loss of NAD-dependent protein deacetylase sirtuin-2 alters mitochondrial protein acetylation and dysregulates mitophagy," Antioxidants \& Redox Signaling, vol. 26, no. 15, pp. 849-863, 2017.

[37] D. B. Lombard, F. W. Alt, H.-L. Cheng et al., "Mammalian Sir2 homolog SIRT3 regulates global mitochondrial lysine acetylation," Molecular and Cellular Biology, vol. 27, no. 24, pp. 8807-8814, 2007.

[38] S. D. Westerheide, J. Anckar, S. M. Stevens Jr., L. Sistonen, and R. I. Morimoto, "Stress-inducible regulation of heat shock factor 1 by the deacetylase SIRT1," Science, vol. 323, no. 5917, pp. 1063-1066, 2009.

[39] S. Gonfloni, V. Iannizzotto, E. Maiani, G. Bellusci, S. Ciccone, and M. Diederich, "P53 and Sirt1: routes of metabolism and genome stability," Biochemical Pharmacology, vol. 92, no. 1, pp. 149-156, 2014.

[40] M. J. Wilking, C. Singh, M. Nihal, W. Zhong, and N. Ahmad, "SIRT1 deacetylase is overexpressed in human melanoma and its small molecule inhibition imparts anti-proliferative response via p53 activation," Archives of Biochemistry and Biophysics, vol. 563, pp. 94-100, 2014.

[41] T. Luo, Histamine H2 Receptor Activation Exacerbates Myocardial Ischemia/Reperfusion Injury by Disturbing Mitochondrial and Endothelial Function, Southern Medical University, Guangzhou, China, 2013.

[42] L. Qi, Y. Tang, Y. You et al., "Comparing the effectiveness of electroacupuncture with different Grades of knee osteoarthritis: a prospective study," Cellular Physiology and Biochemistry, vol. 39, no. 6, pp. 2331-2340, 2016.

[43] Z. Zhang, C. Wang, Q. Li et al., "Electroacupuncture at ST36 accelerates the recovery of gastrointestinal motility after colorectal surgery: a randomised controlled trial," Acupuncture in Medicine, vol. 32, no. 3, pp. 223-226, 2014.

[44] J.-b. Meng, Y.-n. Jiao, G. Zhang et al., "Electroacupuncture improves intestinal dysfunction in septic patients: a 
randomised controlled trial," BioMed Research International, vol. 2018, Article ID 8293594, 9 pages, 2018.

[45] J. B. Yu, S. A. Dong, L. R. Gong et al., "Effect of electroacupuncture at Zusanli (ST36) and Sanyinjiao (SP6) acupoints on adrenocortical function in etomidate anesthesia patients," Medical Science Monitor, vol. 20, pp. 406-412, 2014.

[46] J.-H. Jeon, C.-K. Cho, S.-J. Park et al., "A feasibility study of moxibustion for treating anorexia and improving quality of life in patients with metastatic cancer: a randomized shamcontrolled trial," Integrative Cancer Therapies, vol. 16, no. 1, pp. 118-125, 2017.

[47] R. Torres-Rosas, G. Yehia, G. Peña et al., "Dopamine mediates vagal modulation of the immune system by electroacupuncture," Nature Medicine, vol. 20, no. 3, pp. 291-295, 2014.

[48] C.-Y. Zuo, P. Lv, C.-S. Zhang et al., "Ipsi- and contralateral moxibustion generate similar analgesic effect on inflammatory pain," Evidence-Based Complementary and Alternative Medicine, vol. 2019, Article ID 1807287, 7 pages, 2019.

[49] P.-R. Lv, Y.-S. Su, W. He et al., "Electroacupuncture alleviated referral hindpaw hyperalgesia via suppressing spinal longterm potentiation (LTP) in TNBS-induced colitis rats," Neural Plasticity, vol. 2019, Article ID 2098083, 11 pages, 2019.

[50] J. Yu, S.-x. Yi, X.-r. Chang, R.-d. Yang, and Y.-p. Lin, "Effects of moxibustion on points Zusanli and Liangmen on expression of heat shock protein 70 in different organs of rats," Journal of Traditional Chinese Medicine University of Hunan, vol. 29, no. 4, pp. 67-69, 2009. 